\title{
Parallel and Scalable Precise Clustering
}

\author{
Stuart Byma \\ stuart.byma@epfl.ch \\ EPFL \\ Switzerland
}

\author{
Akash Dhasade \\ akashdhasade@gmail.com \\ IIT Tirupati \\ India
}

\author{
Adrian Altenhoff \\ adrian.altenhoff@inf.ethz.ch \\ ETH Zürich \\ Switzerland
}

\author{
Christophe Dessimoz \\ christophe.dessimoz@unil.ch \\ University of Lausanne \\ Switzerland
}

\author{
James R. Larus \\ james.larus@epfl.ch \\ EPFL \\ Switzerland
}

\begin{abstract}
This paper describes a new technique for parallelizing protein clustering, an important bioinformatics computation for the analysis of protein sequences. Protein clustering identifies groups of proteins that are similar because they share long sequences of similar amino acids. Given a collection of protein sequences, clustering can significantly reduce the computational effort required to identify all similar sequences by avoiding many negative comparisons. The challenge, however, is to build a clustering that misses as few similar sequences (or elements, more generally) as possible.

In this paper, we introduce precise clustering, a property that requires each pair of similar elements to appear together in at least one cluster. We show that transitivity in the data can be leveraged to merge clusters while maintaining a precise clustering, providing a basis for independently forming clusters. This allows us reformulate clustering as a bottom-up merge of independent clusters in a new algorithm called ClusterMerge. ClusterMerge exposes parallelism, enabling fast and scalable implementations.

We apply ClusterMerge to find similar amino acid sequences in a collection of proteins. ClusterMerge identifies $99.8 \%$ of similar pairs found by a full $O\left(n^{2}\right)$ comparison, with only half as many comparisons. More importantly, ClusterMerge is highly amenable to parallel and distributed computation. Our implementation achieves a speedup of 604 times on 768 cores (1400 times faster than a comparable single-threaded clustering implementation), a strong scaling efficiency of $90 \%$, and a weak scaling efficiency of nearly $100 \%$.
\end{abstract}

\section{CCS CONCEPTS}

- Computing methodologies $\rightarrow$ Parallel algorithms; • Applied computing $\rightarrow$ Computational proteomics.

\section{KEYWORDS}

bioinformatics; protein clustering; parallel algorithms

Permission to make digital or hard copies of all or part of this work for personal or classroom use is granted without fee provided that copies are not made or distributed for profit or commercial advantage and that copies bear this notice and the full citation on the first page. Copyrights for components of this work owned by others than the author(s) must be honored. Abstracting with credit is permitted. To copy otherwise, or republish, to post on servers or to redistribute to lists, requires prior specific permission and/or a fee. Request permissions from permissions@acm.org.

PACT '20, October 3-7, 2020, Virtual Event, GA, USA

(c) 2020 Copyright held by the owner/author(s). Publication rights licensed to ACM ACM ISBN 978-1-4503-8075-1/20/10 .\$15.00

https://doi.org/10.1145/3410463.3414646

\section{ACM Reference Format:}

Stuart Byma, Akash Dhasade, Adrian Altenhoff, Christophe Dessimoz, and James R. Larus. 2020. Parallel and Scalable Precise Clustering. In Proceedings of the 2020 International Conference on Parallel Architectures and Compilation Techniques (PACT '20), October 3-7, 2020, Virtual Event, GA, USA. ACM, New York, NY, USA, 12 pages. https://doi.org/10.1145/3410463.3414646

\section{INTRODUCTION}

The ongoing revolution in genome sequencing is generating large and growing datasets whose value is exposed through extensive computation. The high cost of analysis reduces the utility of these databases, particularly when the time for processing increases rapidly as a dataset becomes more inclusive, larger, and more valuable. Asymptotically efficient algorithms are desirable, but sometimes the tradeoff between speed and precision requires less efficient algorithms. In this situation, the time for analysis can be reduced by running on a parallel computer or cluster of computers. This paper describes a new approach to applying parallel computing to protein clustering, an important technique in the field of proteomics, the analysis of the protein sequences contained in an organism's genome.

More specifically, we are interested in finding all similarities among proteins in a given collection. These similarities are important to biological research. For example, similarities among protein sequences are widely used as proxies to infer common ancestry among genes. Similar genes are referred to as homologs, and their detection allows the function of newly sequenced genes to be inferred from well-studied genes since homologs, despite having accumulated substantial differences during evolution, often continue to perform the same biological function. Most of today's molecularlevel biological knowledge comes from the study of a handful of model organisms, which was then extrapolated to other life forms, primarily through homology detection. Several sequence homology techniques are among the 100 most-cited scientific papers of all time [19].

Current approaches to find similar (homologous) proteins are computationally expensive. The baseline is to perform an exhaustive, all-against-all $\left(O\left(n^{2}\right)\right)$ comparison of each sequence against all others. The cost of this is compounded by expensive similarity measures, typically Smith-Waterman (S-W) or another, similarly costly $\left(O\left(n^{2}\right)\right)$ string-matching algorithm. While this approach does find all similar pairs, it scales poorly as the number of proteins increases. Several databases of similar proteins produced this way exist, including OMA [2] and OrthoDB [20]. Building these databases is 
costly. OMA, for example, has consumed over 10 million CPU hours and includes proteins from only 2000 genomes [2].

The large amount of data produced by many laboratories require new methods for homology detection. In a report published in 2014, the Quest for Orthologs consortium, a collaboration of the main cross-species homology databases, reported: "[C]omputing orthologs between all complete proteomes has recently gone from typically a matter of CPU weeks to hundreds of CPU years, and new, faster algorithms and methods are called for" [16]. Ideally, this would be an algorithm with asymptotically better performance that would also find the same similarities as the all-against-all comparison. Unfortunately, fast (sub $O\left(n^{2}\right)$ ) algorithms - based on k-mer counting, sequence identity, or MinHash - find significantly fewer homologs and hence are not suitable for this application. In the absence of a better algorithm, a scalable parallel implementation of an existing solution could help keep pace with the production of sequence data.

One proven technique is to use a clustering algorithm to group similar proteins, followed by all-against-all matching within each cluster to identify the similar pairs. The initial clustering can significantly reduce computation because it avoids a large number of negative comparison results in the full all-against-all computation. Most comparisons are negative, an all-against-all on a test datasets of $60 \mathrm{~K}$ sequences requires 1.8 billion comparisons but finds only 1.2 million similar pairs.

The key, then, is to use a clustering algorithm that will avoid as many of these negative comparisons as possible, while ensuring that similar pairs end up in clusters. Wittwer et al. [21] previously investigated an approach called greedy incremental clustering. In it, clusters are built one sequence at a time, where the first sequence forms a representative of the cluster. Subsequent sequences are processed by comparing them against each representative and adding them to a cluster if the two are similar. If a sequence is not similar to any representative, it becomes the representative of a new cluster.

Crucially, the approach assumes the transitivity of sequence similarity - if $A$ is similar to $B$, and $B$ is similar to $C$, then $A$ will be similar to $C$. Clustering simply extends this definition by assuming that if a sequence is similar to a representative, then it will be similar to all elements in a cluster. Sequence similarity need not be perfectly transitive (as for protein similarity). The published technique takes this into account by using multiple representatives for a cluster. Although it successfully captures up to $99.9 \%$ of similar pairs and reduces computational by $75 \%$, the algorithm is sequential and not easily parallelized. Each sequence depends on the clustered state resulting from all previous sequences, forming a fine-grain synchronization bottleneck and preventing effective scaling.

To solve this problem, we start by reformulating the clustering problem. Our goal was a clustering that ensures that each pair of similar sequences appear together in at least one cluster, which we call a precise clustering. As we will show, however, building a precise clustering solely with sequence similarity is not possible it devolves into an all-against-all computation. To get around this, building on the work of Wittwer et al., we exploited transitivity and distinguished the properties of similarity and a stronger relation transitive similarity. Transitive similarity is defined: if $A$ is transitively similar to $B$, and $A$ is similar to $C$, then $B$ will be similar to $C$. Therefore, if $A$ is a cluster representative, $B$ can be clustered with $A$ and be guaranteed that subsequent similar sequences will also be added to the cluster because they will be similar to $A$. No similarity involving $B$ will be missed, even though there was only one direct comparison involving $B$, thanks to its transitive similarity to $A$. Using an iterative approach, we can guarantee a precise clustering ${ }^{1}$ by clustering each sequence with a transitively similar representative, or, if none exist, giving the sequence its own cluster.

Iterative approaches, however, do not scale. The key to a fast and parallel precise clustering algorithm is that transitive similarity can also be used to merge clusters. If two cluster representatives are transitively similar, the similarity between all members of one cluster and the other cluster's representative can be inferred, and the clusters can be merged. If the two representatives are simply similar, similar members can be exchanged between clusters, maintaining a precise clustering but leaving two clusters. This insight allows us to build clusters in parallel and merge them later, while maintain a precise clustering.

Exploiting this ability to merge clusters, we introduce a new algorithm for precise clustering called ClusterMerge. ClusterMerge places every sequence in a cluster, and then successively merges these clusters until a single set of unmergeable clusters remains. As each individual merge step maintains a precise clustering, the end result is also a precise clustering. When applied to protein similarity, ClusterMerge finds $99.8 \%$ of ground truth similar pairs found by a full all-against-all match.

ClusterMerge exposes a large amount of parallelism in its treelike computation. However, the computation is highly irregular because of the wide span in the length of proteins (hundreds to tens of thousands of amino acids), the $O\left(n^{2}\right)$ string comparison that exaggerates this disparity, and differences in the size of clusters - all of which requires careful dynamic load balancing to achieve good performance. We present efficient parallel and distributed implementations using this cluster merge approach. Our singlenode, shared-memory system scales nearly linearly and achieves a speedup of $21 \times$ on a 24 core machine. Our distributed design achieves a speedup of $604 \times$ while maintaining a strong scaling efficiency of $79 \%$ on a distributed cluster of 768 cores ( $90 \%$ on larger datasets), running $1400 \times$ faster than the incremental greedy clustering of Wittwer et al. [21]. Our distributed implementation exhibits a weak scaling efficiency of nearly $100 \%$ on 768 cores. ClusterMerge and our implementations of protein sequence clustering are opensourced [1]

This paper makes the following contributions:

- A formalization of precise clustering using similarity and transitive similarity.

- An algorithm, ClusterMerge, that reformulates the clustering process in a form that exposes parallelism.

- An application of ClusterMerge to the problem of clustering protein sequences that maintains near-perfect accuracy while achieving high parallel efficiency.

The rest of this paper is organized as follows: 22 reviews related work in clustering and sequence clustering. $\S 3$ formalizes precise clustering and describes in detail both the ClusterMerge algorithm and how it is applied to protein sequences. $§ 4$ discusses

${ }^{1}$ Insofar as the transitive similarity property is accurately determined for the data being clustered. 
our shared memory and distributed implementations of ClusterMerge. $§ 5$ evaluates the algorithm, systems, and their performance in this application. §6 discusses future work and §7 concludes.

\section{RELATED WORK}

Clustering is widely used and has been the subject of considerable research. Cluster techniques used in other fields are difficult to apply to protein clustering, however. Andreopoulos et al. survey uses of the techniques in bioinformatics [3].

Partitioning algorithms generally assume an equivalence relation between elements, which is stronger than the not-necessarily transitive similarity relationship in protein clustering. k-means clustering starts with a target number of clusters, which is unknown in advance for proteins, and partitions the set. Hierarchical methods partition elements into a tree and preserve hierarchy among elements, but generally require a similarity matrix to exist, which is not the case for proteins. They are also computationally expensive $\left(O\left(n^{3}\right)\right)$. Of particular note is agglomerative hierarchical clustering, which also uses bottom-up merge, e.g., ROCK [6]. Density-based clustering uses a local density criterion to locate subspaces in which elements are dense. They can miss elements in sparse regions and generally cannot guarantee a precise clustering. Density-based techniques have received attention from the parallel computing community, with the DBSCAN [15] and OPTICS [4] algorithms being parallelized by Patwary et al. [13, 14].

An additional complication of these methods is that they rely on distance metrics in normed spaces, e.g., Euclidean distance, which are usually inexpensive to compute. The edit distance used for proteins, however, is not a norm and is expensive to compute. Although pure edit distance (i.e., Levenshtein distance) can be embedded in a normed space [12], it is not clear if the gapped alignment necessary for protein similarity can be.

The clustering of biological sequences is the subject of considerable research. Many of the clustering algorithms employ an iterative greedy approach that constructs clusters around representative sequences, one sequence at a time. Some approaches use k-mer counting to approximate similarity (CD-HIT [9], kClust [7], Mash [11]), while others use sequence identity, i.e., the number of exact matching characters (UCLUST [5]). Of note is Linclust [17], an approach that operates in linear time by selecting $m \mathrm{k}$-mers from each sequence and grouping sequences that share a k-mer. The longest sequence in a group is designated its center and other sequences are compared against it, avoiding a great deal of computation.

Unfortunately, sequence identity and k-mers are unsuitable for finding homologs. Protein alignment substitution matrices are heterogeneous (e.g., BLOSUM62 [8]) since distinct amino acids may be closely related. Hence, protein sequences that appear different - with low sequence identity and therefore few or no shared kmers - can often have high alignment scores. These similar pairs will be missed by k-mer-based clustering techniques. For example, the fraction of similar sequence pairs found by kClust, UCLUST, MMSeqs2 linclust, and MMSeqs2 are 10.4\%, 13.5\%, 0.5\%, and 36.4\%, respectively [21].

Wittwer et al. [21] use an iterative greedy approach to cluster protein sequences, using transitivity to avoid comparing each sequence with all others while identifying $\sim 99.9 \%$ of similar pairs.
Our work starts with a similar transitivity function. However, the previous iterative greedy approach is difficult to parallelize because each added sequence depends on the clusters from the previous sequences and requires fine-grained synchronization.

\section{PRECISE CLUSTERING}

A clustering is an arrangement of elements of a set into possibly overlapping subsets (clusters) so that the elements of each subset share a given property. ${ }^{2}$ Precise clustering is a clustering that ensures each pair of elements that share a property appear together in at least one cluster.

For the protein clustering problem, the relationship is the similarity of proteins. After precise clustering, similar proteins can be easily found by pairwise comparison of elements in each of the resulting clusters. Clustering can avoid many dissimilar comparisons, because the dissimilar elements will not appear in the same cluster.

To clustering proteins, we start with a similarity function that produces a measure of the similarity of two proteins. If its value is greater than a threshold, we conclude that the proteins are similar. Otherwise, they are not similar. The only way to determine if two elements are similar is to compare them. We will use $f(i, j)$ to denote the similarity function; $f(i, j)>T$ therefore indicates similarity, where $T$ is a parameter called the threshold. If $f(i, j)>T$, we say that $i$ and $j$ are a significant pair. We assume that the similarity function is reflexive, that is, $f(i, j)>T \Longleftrightarrow f(j, i)>T$. We do not assume that the similarity function satisfies the triangle inequality, that is, $f(i, k)>T \wedge f(k, j)>T \nRightarrow f(i, j)>T$.

Clusters are defined by a single representative element. Every other element in the cluster is similar to the representative. However, cluster members are not necessarily similar to each other. Formally, a cluster $C$ is a subset of elements that has a representative element $r_{C}$ :

$$
\forall e \in C, f\left(e, r_{C}\right)>T
$$

The basic approach to building clusters with these assumptions is to do so greedily and sequentially as with Wittwer's approach [21]. The first element forms the first cluster representative. The next element is compared against the representative of each cluster, and if similar, it is added to the cluster and the comparisons stop. If not similar to any representative, it forms a new cluster with itself as the representative. The process repeats until all elements have been clustered. The problem, however, is that when an element is added to a cluster, it is no longer visible to subsequent comparisons, and elements similar to it, but not to the cluster representative, may not be placed in its cluster. This approach does not produce a precise clustering. An alternative is to form a cluster around a single element, in which case the solution devolves to an all-against-all comparison.

By considering a stronger property than similarity, we can produce a precise clustering as well as avoid unnecessary comparisons. That property is transitive similarity. If element $i$ is transitively similar to an element $j$, the property guarantees that any subsequent element $k$ that is similar to $i$ will also be similar to $j$. If element $i$ is a cluster representative, $j$ can be clustered with $i$ and all elements similar to $j$ will be added to this cluster because of transitive similarity. No significant pairs involving $j$ will be missed, and $j$ does

\footnotetext{
${ }^{2}$ Since an element can be in more than one cluster, clustering is not partitioning.
} 
not need its own cluster. Transitive similarity is not necessarily reflexive (we will discuss why this is the case for protein sequences in Section 3.1). Transitive similarity also implies similarity; if two elements are transitively similar, they are also similar.

More formally, we define a transitivity function $R(i, j)$ to denote transitive similarity between $i$ and $j$.

$$
\forall k, R(i, j) \Longrightarrow f(i, k)>T \wedge f(j, k)>T
$$

$R$ is interpreted as follows: $R(i, j)$ evaluating true implies that if $f(i, k)>T$ then $f(j, k)>T$ for any $k$. That is, if two elements $i, j$ are transitively similar, we know that any third element $k$ that is similar to $i$ will also be similar to $j$. Figure 1 shows a visual representation of transitive similarity between elements $i$ and $j$.

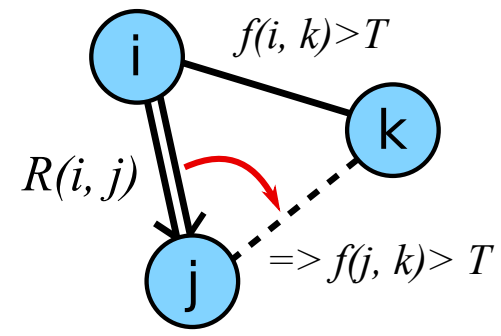

Figure 1: The transitivity function $R(i, j)$ allows an inference that indicates $j$ will be similar to $k$ because $i$ is similar to $k$.

$R$ is required because the similarity function $f(i, j)$ is not guaranteed have a transitive property and we cannot assume that it obeys the triangle inequality.

Most other clustering methods use distances in normed spaces or similarity measures that can be embedded in normed spaces, which obey the triangle inequality (e.g., Euclidean distance). The clustering approach described in this paper handles data that does not have this property, such as clustering multimedia objects (e.g., images) or comparing time series (e.g., stock market, seismological, or climate data). Transitive similarity, as with similarity, is not necessarily inherent in the data. A domain expert must design $R(i, j)$ such that it satisfies the transitive similarity property.

We can modify the greedy sequential algorithm to exploit transitive similarity. First, new elements are tested for transitive similarity (rather than just similarity) with a current cluster's representative. If an element is transitively similar to the representative, it is added to the cluster and can rely on the representative to identify subsequent elements to which it is similar. If the new element is only similar to the representative, the element is added to the cluster but continues to be compared to representatives of other clusters until either 1) a transitively similar one is found or 2) none is found and a new cluster is created for the element. In this way, each element is clustered with those to which it is similar, either by becoming the representative of its own cluster or by relying on a transitively similar representative. This ensures a precise clustering while avoiding comparisons to sequences that are transitively represented.

\subsection{Clustering Proteins}

We will use proteins to illustrate the concepts of similarity, transitive similarity, and clustering, as proteins are the primary motivation for this work. We use the Smith-Waterman (S-W) alignment score as the similarity function for proteins. If the S-W score is above a threshold $T$, two sequences are similar. Figure 2 shows three sequences, $\mathrm{A}, \mathrm{B}$, and $\mathrm{C}$, where $\mathrm{A}$ is similar to $\mathrm{B}$ and $\mathrm{B}$ and similar to C. $\mathrm{C}$, however, is not similar to A because it aligns with a different subsequence of $\mathrm{B}$.

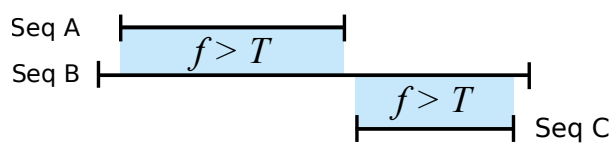

Desired Clustering Result:

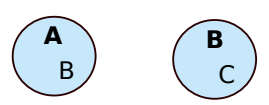

Figure 2: Clustering protein sequences. Sequence $A$ is similar to sequence $B$, which is similar to sequence $C$. $C$ is not similar to A.

If we use only similarity to perform clustering, significant pairs can be missed - the order of evaluation will affect which significant pairs are found. For example, comparing $f(A, B)$ first will lead to a cluster represented by $A$, with $B$ as a member. When $C$ is then compared to the representative $\mathrm{A}$, it is not similar and forms its own cluster. The (B, C) significant pair will be missed. Starting the process with sequence $B$ would lead to a single cluster represented by $\mathrm{B}$, with $\mathrm{A}$ and $\mathrm{C}$ as members.

With transitive similarity, a precise clustering can be formed regardless of comparison order. However, we first need a transitivity function for proteins. Protein sequence alignment does have a transitive property. However, S-W is a local alignment algorithm, meaning that it may not include ("cover") all residues (individual amino acids) in both sequences, especially when the sequences are of different lengths. If a sequence is clustered with a representative that does not completely cover it when aligned, the uncovered subsequence will be unrepresented. This may cause subsequence homologies to be missed, as in the example above.

Therefore, subsequence homologies must be taken into account when designing a transitivity function for proteins. Figure 3 illustrates the transitivity function for proteins using two sequences $\mathrm{X}$ and Y. Depending on the size of each sequence and the alignment, there may be several uncovered residues in each sequence, shown as $\mathbf{u X}$ and $\mathbf{u Y}$ in Figure 3. For one sequence to transitively represent the other, the alignment score between $\mathrm{X}$ and $\mathrm{Y}$ must be greater than $\min T$, the transitivity threshold, a parameter typically greater than $T$. In addition, the number of uncovered residues in each of the sequences must be less than parameter maxU (maximum uncovered), to ensure that homologous subsequences are not missed. In this example, $\mathrm{Y}$ could transitively represent $\mathrm{X}$ because $\mathbf{u X}$ is small, but $\mathrm{X}$ could not transitively represent $\mathrm{Y}$ because a large section of $\mathrm{Y}$ is uncovered. This example also illustrates that $R(i, j)$ for proteins is not reflexive.

Now we return to our example from Figure 2. Following the sequential clustering algorithm, $R(A, B)$ will indicate that $\mathrm{B}$ cannot be transitively represented by $A$, because the alignment does not 


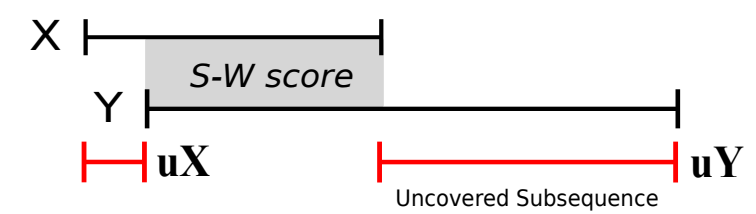

$$
\begin{aligned}
& R(X, Y) \Longrightarrow \text { score }>\min T, u Y<\max U \\
& R(Y, X) \Longrightarrow \text { score }>\min T, u X<\max U
\end{aligned}
$$

Figure 3: Transitivity function for protein sequences.

cover a large subsequence of B. Because they are similar, however, B is added to the cluster with $\mathrm{A}$ and will also represent its own cluster. $\mathrm{C}$ can be transitively represented by $\mathrm{B}$ and will be placed in the cluster with B. We end up with the desired precise clustering shown in Figure 2. With transitive similarity, the order of comparison does not matter - any sequence not transitively represented by an existing cluster will represent its own cluster.

\subsection{The ClusterMerge Algorithm}

While the greedy algorithm studied in the previous subsections is useful in understanding transitive similarity and precise clustering, it is sequential and difficult to parallelize because processing each element depends on the clustered state of all previous elements, forming a synchronization bottleneck. To solve this, we introduce ClusterMerge, a new algorithm for precise clustering that exposes parallelism by structuring the clustering computation as a bottomup merge of clusters.

3.2.1 Merging Clusters. The key to exposing parallelism lies in recognizing that clusters with transitively similar representatives can be merged. This allows us to reframe clustering as a series of cluster merges. Two clusters can be merged as follows. First, their representatives are compared with the similarity function $f$. If they are similar, the transitivity function $R$ is applied to see if they are transitively similar. If so, the clusters can be combined into a single cluster, with one representative for all elements. Otherwise, if the representatives are similar but not transitively similar, members of either cluster might be similar to the other representative. ${ }^{3}$ To avoid missing these, each cluster's elements are compared against the other's representative and the similar elements are added to the other cluster. Finally, if the representatives are not similar, both clusters remain unchanged. The result is a set of one merged cluster or a set of two clusters whose representatives are not transitively similar and thus not mergeable.

Merging can also be applied to two sets of clusters. Algorithm 1 describes the process in detail. Each cluster in the first set (cs1) is compared to and possibly merged with every cluster in the second set (cs2). For each cluster pair, the process described above is applied. Finally, all un-mergeable clusters are returned in a new set.

\footnotetext{
${ }^{3}$ Note that we are assuming these two clusters were computed independently, not sequentially as above.
}
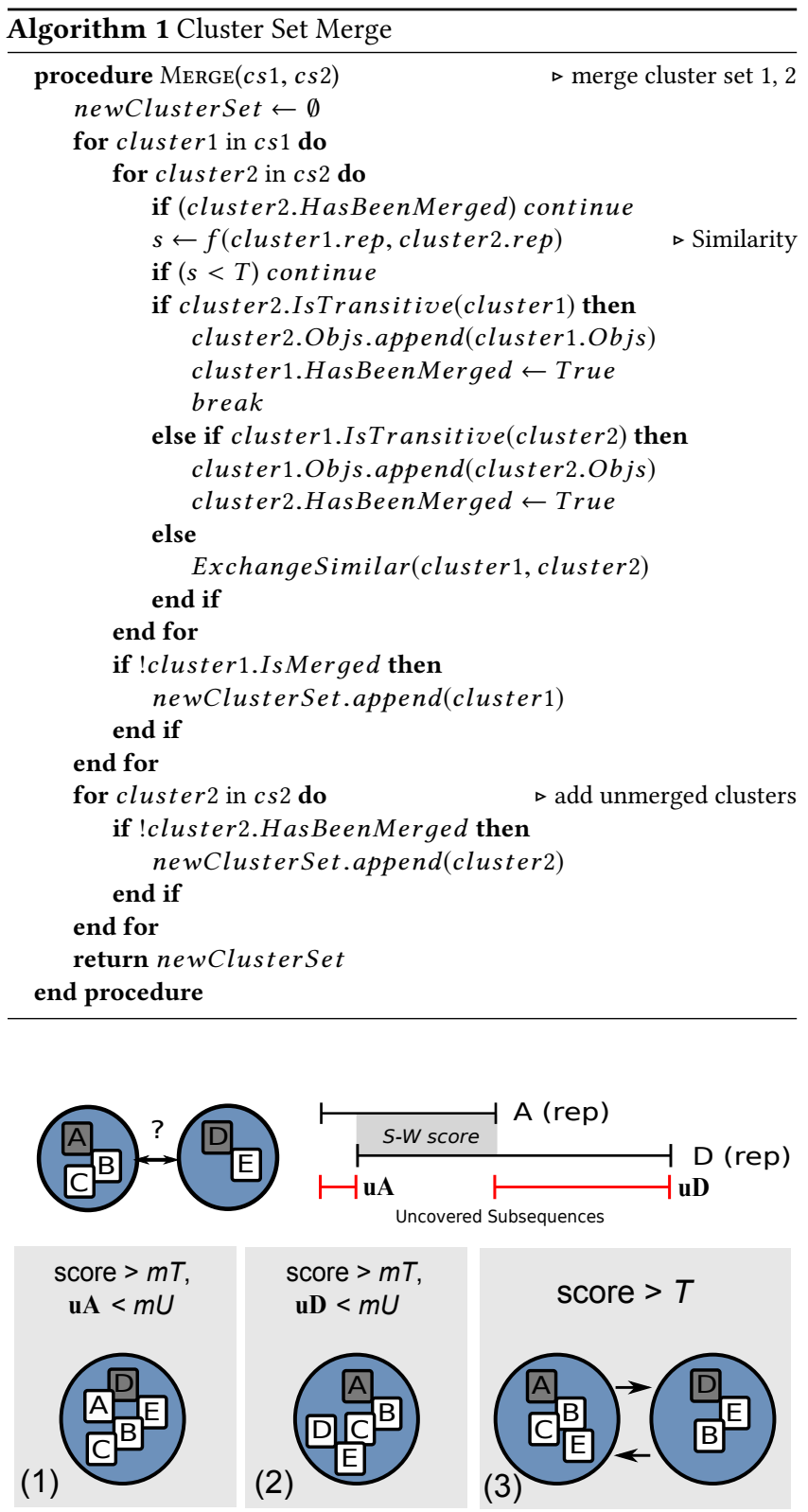

Figure 4: Potential outcomes of merging two clusters of proteins.

Figure 4 illustrates the possible results of merging two clusters of proteins using this algorithm. Sequences $\mathrm{X}$ and $\mathrm{Y}$ are representatives of two clusters. Based on the result of the transitivity function (described above in Section 3.1) applied to X and Y, either 1) the cluster of $\mathrm{X}$ is merged into the cluster of $\mathrm{Y}, 2$ ) the cluster of $\mathrm{Y}$ is merged into the cluster of $X$ or 3 ) the clusters exchange similar members if $\mathrm{X}$ and $\mathrm{Y}$ are similar but not transitively similar. This results in a set of one fully merged cluster as in situations (1) and (2), or two clusters whose representatives are not transitively similar (situation 3). 


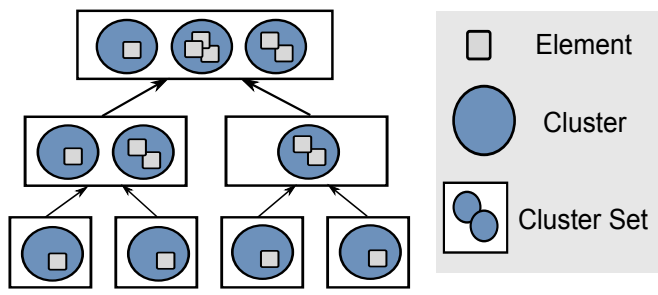

Figure 5: ClusterMerge algorithm. Elements are placed in trivial clusters which are then merged until an unmergeable set remains.

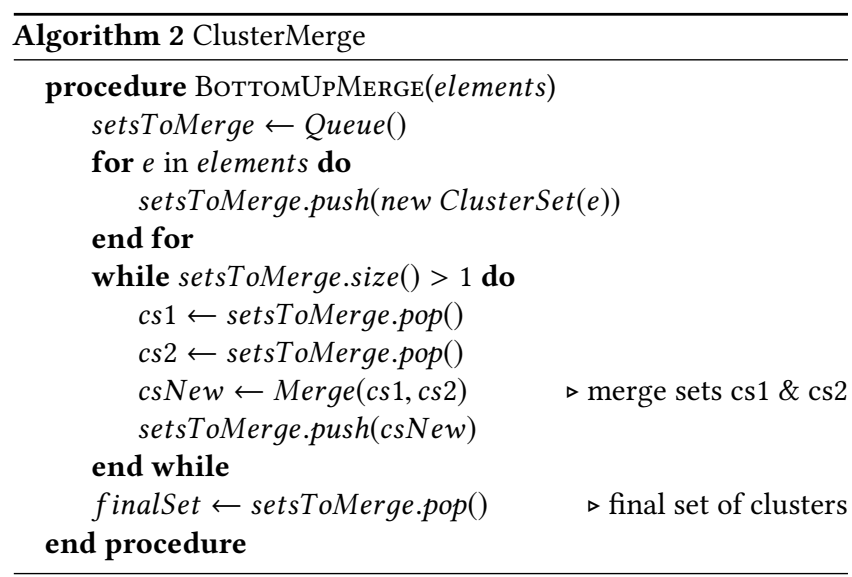

3.2.2 ClusterMerge Algorithm. The ClusterMerge algorithm uses cluster merging to perform precise clustering. Each element is initially placed in its own cluster as its representative and each cluster is placed in its own set. Algorithm 1 is then applied to merge cluster sets in a bottom-up fashion as depicted in Figure 5 .

Algorithm 2 describes this bottom-up merge process. To start, a new cluster set is created for each element. These cluster sets are added to a FIFO queue of sets to merge (the set merge queue). The algorithm pops two sets off the queue, merges them using Algorithm 1, and pushes the resulting cluster set onto the queue. The process terminates when only one set is left. This algorithm forms the basis of the ClusterMerge implementations further described in Section 4.

3.2.3 Discussion. With a complete ${ }^{4}$ transitivity function, ClusterMerge will not miss any similar element pairs because all elements are implicitly compared against each other, either directly or implicitly via a transitive representative. The chosen element remains representative of its cluster until it is (possibly) fully merged with another cluster. After that, transitivity ensures that subsequent similar elements will then also be similar to the new representative. Therefore, even though cluster members are not necessarily transitively represented by the cluster representative, the algorithm also ensures that those non-transitively similar elements retain their own cluster.

In reality, a complete and computationally efficient transitivity function rarely exists for non-trivial elements, so an approximation

\footnotetext{
${ }^{4} \mathrm{~A}$ complete transitivity function correctly captures all transitive similarity in the data
}

is necessary, as in our motivating example of protein sequence clustering. Incompleteness in the transitivity function can lead ClusterMerge to miss some significant pairs. However, as is demonstrated in Section 5, even an approximate transitivity function can produce very good results. This is also why the transitivity function is applied both ways in Algorithm 1 - approximate transitivity is not necessarily reflexive.

The threshold value $T$ is a parameter that would be chosen by an end end-user domain expert to specify the desired degree of similarity between elements. Users do not currently have influence over which elements are used as representatives, which are selected by the algorithm.

\subsection{Complexity}

The worst-case complexity of ClusterMerge is $O\left(n^{2}\right)$, however this is a fairly strict upper bound. Consider the tree structure formed by the cluster set merges, which has a depth of $\log _{2} n$, where $n$ is the number of elements to be clustered. At the first layer, there are $n / 2$ merges possible, each comparing two clusters of one element each. At there second layer, there are $n / 4$ merges, each comparing a worst-case total of 4 clusters (if no full clusters were merged in the layer above). Generalizing this pattern we obtain

$$
n / 2 \times 1^{2}+n / 4 \times 2^{2}+n / 8 \times 4^{2} \ldots
$$

which we can reduce to

$$
2 n \sum_{i=0}^{\log _{2} n} 2^{i}=2 n \cdot 2^{\log _{2} n+1}-1=2 n \cdot(2 n)-1 \approx n^{2}
$$

However, when clusters are fully merged, there is a reduction in work at each level, leading to sub- $n^{2}$ performance. In a more optimal case, assuming that at each step the merger of two cluster sets cuts the total number of clusters in half, complexity falls to $O(n \log n)$. Actual complexity therefore depends on the amount of transitivity in the data being clustered.

\section{PARALLEL CLUSTERMERGE}

There are several opportunities for parallelism inherent in ClusterMerge, which we will use to construct efficient systems for both shared-memory and distributed environments. Since the designs for shared-memory and distributed systems differ slightly, we will refer to the shared-memory design as Shared-CM and the distributed design as Dist-CM.

The obvious parallelism in ClusterMerge is that smaller sets near the bottom of the tree can be merged in parallel. In general, as long as there are sets of clusters in the setsToMerge queue, threads can pop two sets, merge them, and push the result back onto the queue. These operations are independent and can be processed in parallel.

However, after many merges, only a few large sets will remain. The "tree-level" parallelism is no longer sufficient to keep system resources occupied, and, in fact, the final set merge is always sequential. Therefore, merges of individual sets must be parallelized, which is also valuable because the sets can grow to be very large.

Shared-CM and Dist-CM both use the same technique to split large set merges into smaller work items called partial merges. Consider merging two cluster sets, Set 1 and Set 2 (Figure 6). A partial 

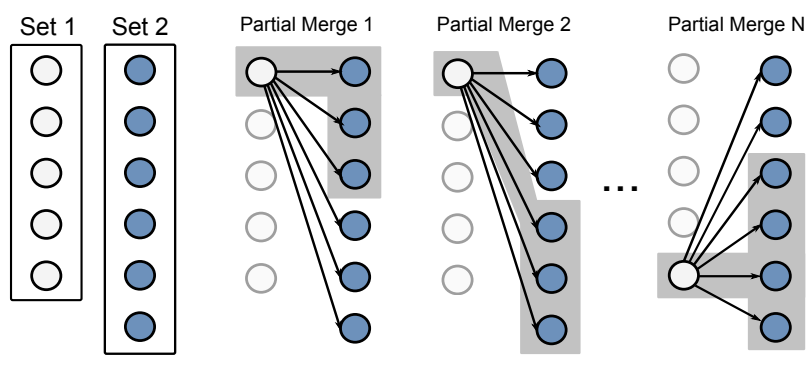

Figure 6: A merge of two large cluster sets is split into partial merges. Threads (or remote workers) can then simultaneously process a merge of two sets.

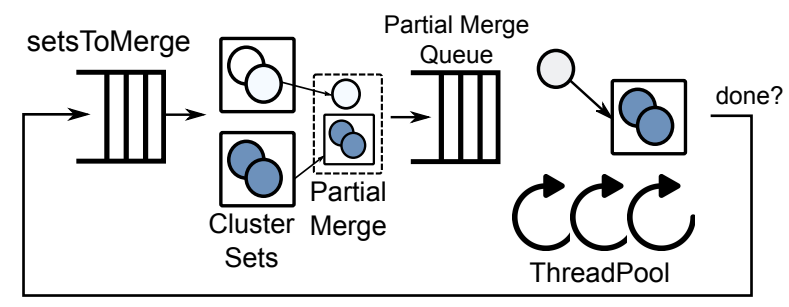

Figure 7: High-level architecture of Shared-CM.

merge merges a single cluster from Set 1 into a subset of the clusters of Set 2. Threads or remote workers can execute these partial merges in parallel by running the full inner loop of Algorithm 1. This allows the system to maintain a consistent work granularity by scheduling a similar number of element comparisons in each partial merge. The load is then evenly balanced, preventing stragglers and leading to better efficiency. Shared-CM and Dist-CM differ only in how they coordinate the synchronization of the results of partial merges.

\subsection{Shared-Memory}

Shared-CM is designed to be run on a commodity multicore computer. Shared-CM splits set merges into partial merges as described above and allows threads to update the clusters in each set in place.

Consider a thread executing a partial merge, where a cluster from Set 1 is being merged into some clusters in Set 2. While our thread has exclusive access to the cluster from Set 1, it has no such guarantee for the clusters in Set 2. Concurrent modifications, including removal of clusters and creation of new ones, can happen because of partial merges of other items from Set 1 .

Shared-CM uses locking to prevent races. The merging logic is the same as in Algorithm 1, however clusters of the second set are locked before being modified in-place. The final merged set is simply the remaining clusters of Set 1 and Set 2 that have not been fully merged. Ordering is not guaranteed and the process sacrifices determinism, but the significant pair recall is the same as a deterministic execution.

Figure 7 illustrates the system design. A coordinating thread pops two sets off the setsToMerge queue. The merge is divided into partial merges as described above, which are inserted into a partial merge queue. A pool of worker threads then process the partial merges. Once the partial merges for a set merge are completed, the coordinating thread collects remaining clusters from both sets into a merged set and pushes it onto the queue.

As long as there are sets remaining to be merged, partial merges can be scheduled and all processors on the machine kept busy. Multiple cluster set merges can also be split into partial merges and executed simultaneously. Shared-CM can scale nearly linearly across cores, with full experimental results detailed in $\S 5.2$.

\subsection{Distributed}

While locking works well in a multicore computer, it would limit scalability on a distributed cluster. Instead, Dist-CM ensures that any processing sent to remote workers is fully independent. Workers therefore have no communication with each other and only communicate with a central controller, resulting in a very scalable system. Dist-CM is a controller-agent distributed system. The controller is responsible for managing the shared state of the computation, while the majority of the computing is performed by remote workers.

Dist-CM uses several techniques to control the size of an average work item to prevent load imbalance and enable efficient scaling. First, batching is used to group small cluster sets together as a single work item. This provides each remote worker with a unit of computation that will not be dwarfed by communication overhead. Batches are executed by a remote worker, and the resulting cluster set is returned to the controller and pushed back into the setsToMerge queue. Batching is important for the early phase of computation, where each set is small and requires little computation.

For larger merges near the top of the tree, Dist-CM uses partial merges in much the same manner as Shared-CM to maintain a consistent work item granularity. Because there is no inter-worker communication, the controller is responsible for managing partial merge results as they are returned. Recall that each partial merge work item merges a single cluster from Set 1 into a subset of clusters of Set 2. The result of a partial merge executed by a remote worker is then a set containing some clusters of Set 2, with the single cluster from Set 1 possibly fully merged with one of them and/or some elements exchanged with some clusters. If the single cluster was not fully merged, it will be included in the returned set.

For each outstanding merger of two cluster sets, the controller maintains a partially merged state of the final result, identified by an ID associated with all partial merges involved in its computation. This partially merged state begins as simply both sets of clusters. When a partial merge result is returned to the controller, it uses the ID to look up the associated partially merged state. The controller will then update the partially merged state with the results of the returned partial merge, adding any elements to existing clusters and marking any fully merged clusters. After processing the final partial merge for a given set merge, the merge is complete and the resulting set is constructed by simply combining non-fully merged clusters from both sets.

Figure 8 depicts the design of Dist-CM. Once again the setsToMerge queue is loaded with single element cluster sets, but at the central controller. A coordinating thread on the controller will pop two sets off the queue to merge together. If the sets (in terms 


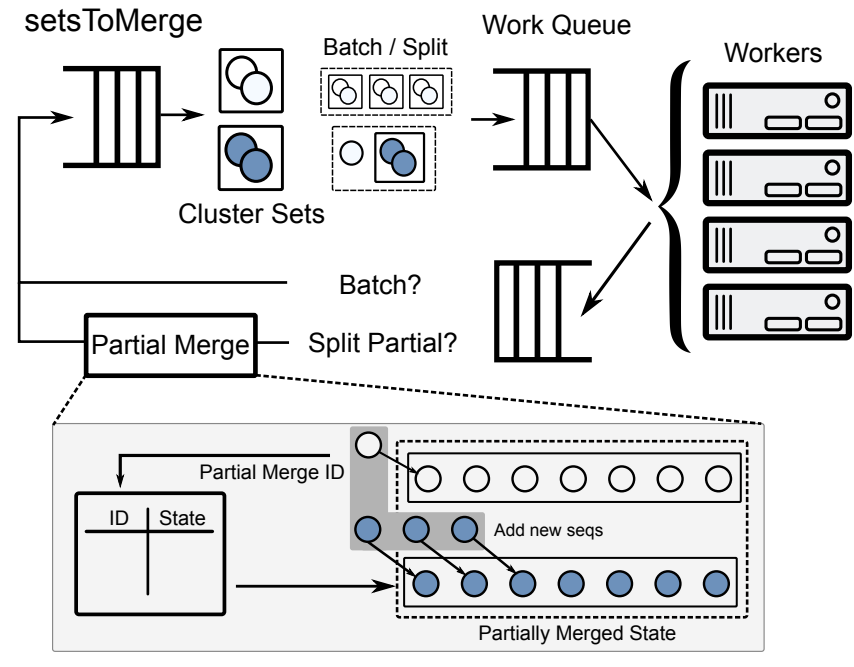

Figure 8: High-level architecture of Dist-CM.

of total clusters) are smaller than a batch size parameter, the thread will pop more sets until it has sets whose total number of clusters is equal to or greater than the batch size parameter. These sets are compiled into a batch work item and pushed into a central work queue. If the sets popped by the coordinating thread are large, they are split into partial merges, with the number of sequences in each cluster taken into account to evenly size each request. This dynamic load balancing keeps straggling in remote workers to a minimum, and is important in achieving good scaling. Partial merges are then are pushed into the central work queue as individual work items. The central work queue feeds a set of remote worker nodes.

Results from workers are returned to the controller and either pushed back to the setsToMerge queue if a batch result (which is a complete cluster set), or used to update an associated partially merged state if it was a partial merge result. If the partially merged state was completed by the work item in question, the now-complete set is pushed to the setsToMerge queue. The process is complete when the final set of the merge tree is complete.

The trade-off inherent in this design is that Dist-CM does more work than necessary in exchange for zero communication among workers. A cluster in a partial merge will continue to be merged into clusters in the set by Dist-CM even if they were fully merged away in other workers. As a result, Dist-CM can perform slightly more work than Shared-CM and can occasionally add the same elements to the same cluster (these duplicates are easily removed by the controller). This trade-off leads to Dist-CM being about $17 \%$ slower than Shared-CM when using a single remote worker in our application of protein sequence clustering.

Scalability can be adversely affected by average latency or amount of work in a single work item. Very small work items will have communication overheads that may exceed the actual computation. Very large work items can cause stragglers and load imbalance that can leave processors idle. Early versions of Dist-CM operated without dynamic sizing of partial merges; each one merged a single cluster into the entirety of the other set. This led to massive load imbalance and long idle periods as large merges were completed.
Dynamic sizing of partial merges was crucial to ensure proper load balance and minimize stragglers, improving scaling efficiency by almost $3 \times$.

Furthermore, unbalanced work distribution can cause stragglers as well, if some workers locally queue more work than others. To avoid this, we switched from a round-robin work distribution method to a Join-Idle-Queue [10] approach in which workers inform the controller when they need more work. This keeps all workers busy so long as work is available while limiting workerlocal queuing.

\subsection{Optimizations}

Several important optimizations enable efficient scaling of Dist$\mathrm{CM}$. In early versions, the controller sent whole sets with each partial merge, which nearly saturated available network bandwidth. Communication overhead was greatly reduced through several techniques. First, each worker replicates the sequence dataset and refers to sequences by a 4-byte index. Actual sequence data is never transferred, and even large clusters with thousands of sequences only require a few kilobytes. Second, one of the sets in a series of partial merges is cached on each worker, so it is only transferred over the network once. Finally, the results of partial merge are returned as diffs, i.e., only newly added sequences in each cluster.

\section{EVALUATION}

We evaluate several aspects of ClusterMerge and its implementations applied to protein sequence clustering. Both Shared-CM and Dist-CM variants are evaluated in this section. Both implementations are written in $\mathrm{C}++$ and compiled with GCC 5.4.0. To compute sequence similarities, we use the Smith-Waterman library SWPS3 [18].

We use two datasets for our evaluation. One is a dataset of 13 bacterial genomes extracted from the OMA database [2], a total of 59013 protein sequences ( $59 \mathrm{~K}$ dataset). This is the same dataset used by Wittwer et al., which allows comparison with their implementation. The second dataset is a large set of eight genomes from the QfO benchmark totaling 90557 sequences ( $90 \mathrm{~K}$ dataset). Although these are a small fraction of the available databases, each represents billions of possible similar pairs, taking many hours to evaluate in a brute-force manner.

Our tests are performed using servers containing two Intel Xeon E5-2680v3 running at $2.5 \mathrm{GHz}$ (12 physical cores in two sockets, 48 hyperthreads total), 256 GB of RAM, running Ubuntu Linux 16.04. The distributed compute cluster consists of 32 servers (768 cores), a subset of a larger, shared deployment. These are connected via $10 \mathrm{~Gb}$ uplinks to a $40 \mathrm{GbE}$-based IP fabric with 8 top-of-rack switches and 3 spine switches. The dataset is small enough such that a local copy can be stored on each server. In fact, even large protein datasets are easily stored on modern servers. For example, the complete OMA database of 14 million protein sequences fits within 10GB, a fraction of modern server memory capacity.

Our baseline for clustering comparisons is the incremental greedy precise clustering of [21], which is the only clustering method that can achieve an equivalent level of similar pair recall. 


\subsection{Clustering and Similar Pair Recall}

For consistency, our clustering threshold is the same as the incremental greedy precise clustering in Wittwer et al. [21], a SmithWaterman score of 181 . The threshold is low, but this is necessary to find distant homologs. After ClusterMerge identifies clusters, an intra-cluster all-against-all comparison is performed, in which the sequence pairs within a cluster are aligned using Smith-Waterman. Those with a score higher than the clustering threshold are recorded as a similar pair. For our datasets, the number of actual similar pairs is small compared to the number of potential similar pairs (e.g. 1.2 million actual out of 1.74 billion potential), leading to relatively few alignments to complete this stage. Biologists may perform additional alignments to derive an optimal alignment with respect to different scoring matrices, however this is orthogonal to the concerns of this paper.

Recall is the percentage of ground truth pairs found by our systems. The ideal recall is $100 \%$. Both Shared-CM and Dist-CM ClusterMerge, using a minimum full merge score $(m T)$ of 250 and a max uncovered residues $(m U)$ of 15 , produce clusters with a recall of $99.8 \pm 0.01 \%$. Recall variability is negligible and is due to the non-determinism of parallel execution. Of the pairs missed by ClusterMerge, very few were high scoring pairs. The median score of a missed pair is 191 and the average score of a missed pair is 235. These values are very close to the cluster threshold itself (in contrast to high scoring pairs, which can be greater than 1000), indicating that these are not likely biologically "important" pairs (Figure 9). ClusterMerge misses only a handful of high scoring pairs, around one-millionth of total significant pairs, as seen in Figure 9.

In clustering the $59 \mathrm{~K}$ sequence dataset, ClusterMerge performs approximately 871 million comparisons. By contrast, the full, allagainst-all comparison requires approximately 1.74 billion comparisons, showing that ClusterMerge reduces comparisons by nearly $50 \%$. This measurement is not the same as computation time reduction used by Wittwer et al., as computation time is not directly proportional to the number of computations. The computation time of a single comparison, depending on the length of the sequences, can vary by several orders of magnitude. As a result, Shared-CM is faster by wall-clock time than greedy incremental clustering when using a single core ( $\$ 5.2)$. This is also due to a combination of efficient implementation and language choice ( $\mathrm{C}++$ vs. Python).

In terms of the clusters themselves, ClusterMerge generates a similar clustering profile as incremental greedy clustering [21], with a total of 33,562 clusters. In each, the vast majority of clusters contain between 1 and 4 sequences, with a few large clusters (33\% of clusters contain more than 10 sequences, $8 \%$ of clusters contain more than 100 sequences, $0.5 \%$ of clusters contain more than 1000 sequences). ClusterMerge generates slightly larger outliers, with its largest cluster containing approximately 1500 sequences as opposed to the greedy method's largest cluster of around 1150 sequences.

Figure 9 shows that ClusterMerge and our transitivity function are relatively insensitive to parameter variations. Lower clustering thresholds $T$ and lower full merge thresholds $m T$ generally lower the number of missed similar pairs, although the absolute percentage of missed pairs remains extremely low, with the majority being low-scoring pairs.

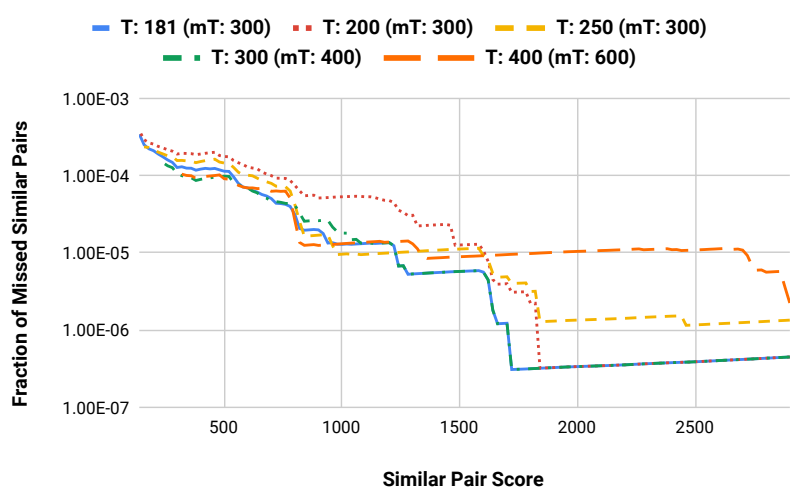

Figure 9: Cumulative fraction of missed pairs reaching at least a certain similarity score, as the clustering threshold $T$ and fully merge threshold $m T$ are varied $(m U=15)$. ClusterMerge shows a low sensitivity to small parameter variations, while most missed similar pairs remain low-scoring ones.

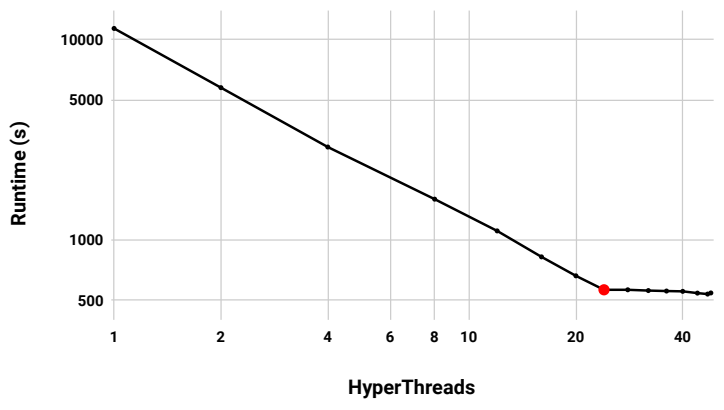

Figure 10: Scaling of Shared-CM up to 48 threads. Scaling is nearly linear up to all 24 physical cores, while hyperthreading provides no benefit.

\subsection{Multicore Shared-Memory Performance}

In this section, we evaluate how well Shared-CM performs on a single multicore node. This experiment uses a reduced dataset of 28600 sequences, to reduce runtimes at low thread counts. Figure 10 shows the total runtime decreases as we increase the number of threads. Shared-CM achieves near-linear scaling - profiling with Intel VTune indicates little or no lock contention. Memory access latency and NUMA have no effect as the workload is computebound.

Note, however, that scaling is linear only on physical cores. The primary compute bottleneck is the process of aligning representative sequences using Smith-Waterman, which processes data that fits in the L1 cache and is able to saturate functional units with a single thread. Therefore, hyperthreading provides no benefit.

The only major impediment to perfect scaling is some loss of parallelism before the last and second-last merges, since the secondlast merge must be fully completed before work for the last merge can start to be scheduled. 
Shared-CM with a single thread clusters the bacteria dataset in 31905 seconds, compared to 1486 seconds with 24 threads, a speedup of $21.5 \times$. To compare with incremental greedy clustering, we run Wittwer's single-threaded code [21] on our machine with the same dataset, resulting in a runtime of 89486 seconds. SharedCM is approximately $2.8 \times$ faster on a single core, and $60.2 \times$ faster using all cores.

\subsection{Distributed Performance}

Dist-CM allows us to scale ClusterMerge beyond a single server. To evaluate the scaling of Dist-CM, we hold the dataset size constant and vary the number of servers used to process work items (batches or partial merges), otherwise known as strong scaling. The baseline single core runtime for Dist-CM clustering the 59K dataset is 39314 seconds. Figure 11a shows that on 32 nodes (768 cores) Dist-CM clusters the dataset in 65 seconds, resulting in a speedup of $604 \times$. Strong scaling efficiency at 768 cores is $79 \%$. Compared to singlethreaded incremental greedy clustering [21], Dist-CM is $2.27 \times$ faster using a single core, and $1400 \times$ faster using the full compute cluster.

The reason for sub-linear scaling is essentially the same as with Shared-CM - around the last few merges of cluster sets, work scheduling may halt as the system waits for an earlier merge to finish before being able to schedule more work. There will always be some small portion of sequential execution, so perfect scaling is impossible by Amdahl's Law.

That being said, this sequential section is proportionally lower with larger datasets. Figure 11b shows Dist-CM strong scaling when clustering the larger $90 \mathrm{~K}$ sequence dataset. The scaling is much more efficient ( $90 \%$ at 32 nodes), with a speedup of $28.7 \times$ relative to one server node.

In addition, we perform a weak scaling experiment in which we vary the amount of work in proportion to the number of nodes. Because our dataset is evolutionarily diverse and has relatively low levels of transitivity, ClusterMerge is closer to $O\left(n^{2}\right)$ in the number of sequences. The amount of actual work increases quadratically with the number of sequences. Figure 12 clearly shows this by varying the number of sequences that Dist-CM clusters using 10 worker nodes. The runtime curve fits almost exactly to a degree two polynomial. Therefore, for our weak scaling experiment, we vary the number of sequences at each step by a square root factor to maintain a proportional increase in workload. Figure 13 shows the results, again while clustering using 1 to 32 nodes. Runtime remains nearly constant throughout, indicating a weak scaling efficiency of 95-100\%. We thus expect that Dist-CM will be able to cluster much larger datasets while maintaining high scaling efficiency.

\subsection{Effect of Dataset Composition}

As noted in section $\$ 3.3$, complexity and therefore runtime depend on how many clusters can be fully merged at each level of the tree. If the transitivity function accurately represents similar elements, the number of full merges at each level is primarily affected by the number of transitively similar elements in a dataset. More transitively similar elements will result in more complete cluster merges, bringing runtime complexity closer to the $O(n \log n)$ optimum.

For protein clustering, the dataset with 13 bacterial genomes has a relatively low number of transitively similar sequences since the

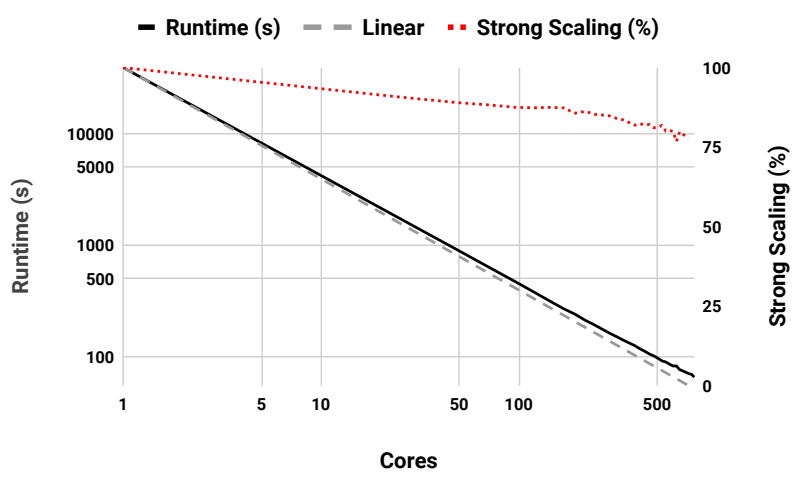

(a) $59 \mathrm{~K}$ dataset, $79 \%$ scaling efficiency.

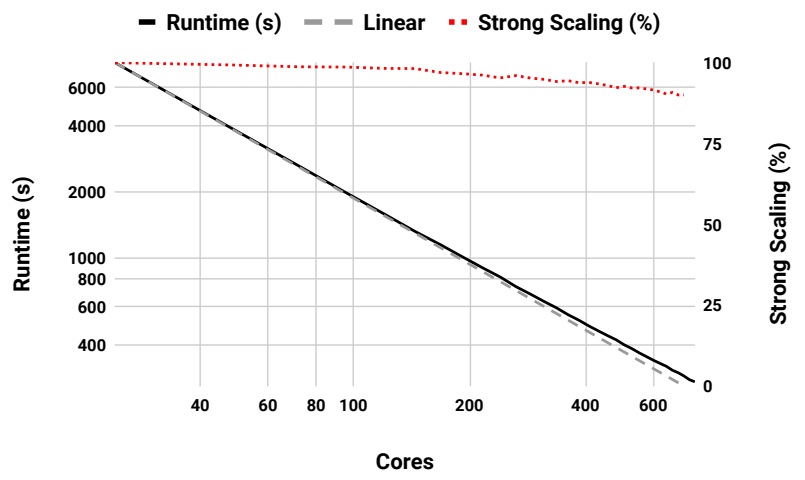

(b) $90 \mathrm{~K}$ dataset, $90 \%$ scaling efficiency.

Figure 11: Scaling of Dist-CM over 32 servers (768 cores).

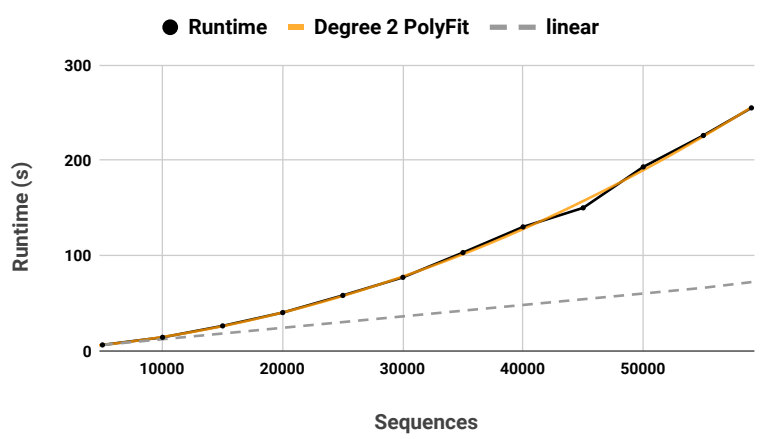

Figure 12: Workload scaling of Dist-CM.

species are genetically very distant (more distant than humans and plants). Given a set of more closely related genomes, with more transitively similar sequences, we would expect ClusterMerge to generate fewer clusters and run much faster. To test this hypothesis, we clustered a third dataset of more closely related Streptococcus bacteria genomes, consisting of 33 genomes (69648 sequences, similar to the other dataset). 


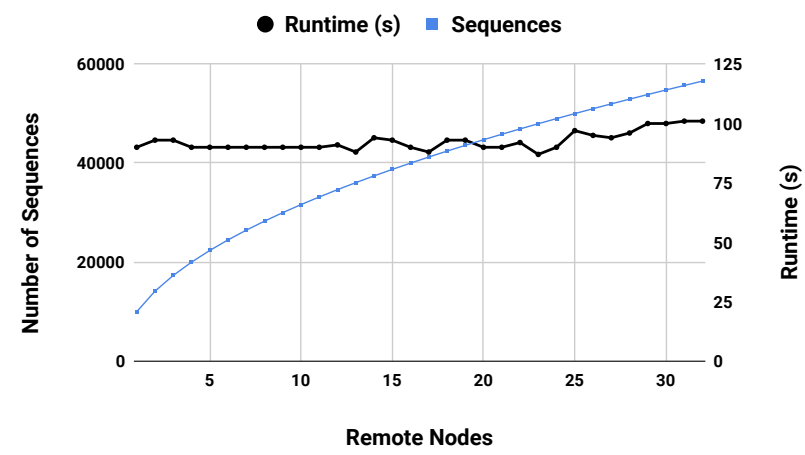

Figure 13: Dist-CM weak scaling over a 32 node (768 core) cluster. Nearly $100 \%$ efficiency at 32 nodes.

Using Shared-CM with 48 threads, the clustering is completed in 283 seconds, producing 10500 clusters. As predicted, clustering is much faster than the 13 bacterial genome dataset (1486 seconds) as the number of clusters is much lower. In addition, ClusterMerge again produced a high recall of $99.7 \%$ of similar pairs relative to a full all-against-all.

\section{FUTURE WORK}

Both of our implementations, Shared-CM and Dist-CM, perform and scale well. However many improvements are possible. Dataset size may expose limits to the current implementation. Very large clusters may produce work items that are still too large, which may cause straggling. Additional splitting beyond the current partial merge may be necessary. Extreme imbalance in cluster sizes between two sets to be merged may also require more creative scheduling of partial merges to avoid large variation in work item size.

Larger datasets also pose a challenge for highly sensitive parameter settings for similarity and transitive similarity, like the ones used in our current evaluation. High sensitivity parameters mean that even distantly related sequences will be seen as similar and placed together in clusters. A consequence is that some clusters can grow quite large, and clusters can contain large overlaps in the sequence sets they contain. One remedy for this is to use less sensitive parameters, and forgo detecting those more distantly related sequences. The alternative is to find a new method or enhancement to ClusterMerge that can reduce the amount of sequence set overlap between clusters while maintaining sensitivity.

For our application to proteins, the current computational bottleneck is the Smith-Waterman alignment function. Runtimes could be improved with a more efficient S-W implementation. We are actively investigating protein alignment-friendly S-W hardware implementations. Similarly, more approximate or less precise alignment methods could be used, though this may come at the cost of precision.

GPUs may also be used to accelerate the similarity and transitivity comparisons. It may also be worth investigating if the ClusterMerge algorithm itself exhibits the memory access patterns suitable for GPUs, however it is unclear if the intensity of SIMD operations within ClusterMerge is high enough to effectively take advantage of GPUs.

\section{CONCLUSION}

ClusterMerge is a parallel and scalable algorithm for precise clustering of elements. When applied to protein sequences, ClusterMerge produces clusters that encompass $99.8 \%$ of significant pairs found by a full all-against-all comparison, while performing $50 \%$ fewer similarity comparisons. Our implementations achieve speedups of $21.5 \times$ on a 24 -core shared-memory machine and $604 \times$ on a cluster of 32 nodes (768 cores). The distributed implementation of ClusterMerge for protein clustering can produce clusters $1400 \times$ faster than a single-threaded greedy incremental approach. ClusterMerge is open source and available [1].

We hope that ClusterMerge will help to form a comprehensive "map" of protein sequences. In theory, clustering could proceed to a point where any given new protein sequence would be represented completely by a subset of existing clusters. No new clusters would need to be added, and any new protein could be classified in $O(n \log n)$ time only. However, it is not yet clear how many different genomes would be required to form such a map.

\section{REFERENCES}

[1] 2020. ClusterMerge Repository. https://github.com/epfl-vlsc/clustermerge

[2] Adrian M. Altenhoff, Natasha M. Glover, Clément-Marie Train, Klara Kaleb, Alex Warwick Vesztrocy, David Dylus, Tarcisio M. de Farias, Karina Zile, Charles Stevenson, Jiao Long, Henning Redestig, Gaston H. Gonnet, and Christophe Dessimoz. 2018. The OMA orthology database in 2018: retrieving evolutionary relationships among all domains of life through richer web and programmatic interfaces. Nucleic Acids Research 46, D1 (Jan. 2018), D477-D485. https://doi. org/10.1093/nar/gkx1019

[3] Bill Andreopoulos, Aijun An, Xiaogang Wang, and Michael Schroeder. 2009. A roadmap of clustering algorithms: finding a match for a biomedical application. Briefings in Bioinformatics 10, 3 (May 2009), 297-314. https://doi.org/10.1093/ $\mathrm{bib} / \mathrm{bbn} 058$

[4] Mihael Ankerst, Markus M. Breunig, Hans-Peter Kriegel, and Jörg Sander. 1999. OPTICS: Ordering Points to Identify the Clustering Structure. In Proceedings of the 1999 ACM SIGMOD International Conference on Management of Data (SIGMOD '99). ACM, New York, NY, USA, 49-60. https://doi.org/10.1145/304182.304187 event-place: Philadelphia, Pennsylvania, USA.

[5] Robert C. Edgar. 2010. Search and clustering orders of magnitude faster than BLAST. Bioinformatics 26, 19 (Oct. 2010), 2460-2461. https://doi.org/10.1093/ bioinformatics/btq461

[6] Sudipto Guha, Rajeev Rastogi, and Kyuseok Shim. 2000. Rock: A robust clustering algorithm for categorical attributes. Information Systems 25, 5 (July 2000), 345-366. https://doi.org/10.1016/S0306-4379(00)00022-3

[7] Maria Hauser, Christian E. Mayer, and Johannes Söding. 2013. kClust: fast and sensitive clustering of large protein sequence databases. BMC Bioinformatics 14, 1 (Aug. 2013), 248. https://doi.org/10.1186/1471-2105-14-248

[8] S Henikoff and J G Henikoff. 1992. Amino acid substitution matrices from protein blocks. Proceedings of the National Academy of Sciences of the United States of America 89, 22 (Nov. 1992), 10915-10919. https://www.ncbi.nlm.nih.gov/pmc/ articles/PMC50453/

[9] Weizhong Li and Adam Godzik. 2006. Cd-hit: a fast program for clustering and comparing large sets of protein or nucleotide sequences. Bioinformatics (Oxford, England) 22, 13 (July 2006), 1658-1659. https://doi.org/10.1093/bioinformatics/ btl158

[10] Yi Lu, Qiaomin Xie, Gabriel Kliot, Alan Geller, James R. Larus, and Albert Greenberg. 2011. Join-Idle-Queue: A novel load balancing algorithm for dynamically scalable web services. Performance Evaluation 68, 11 (Nov. 2011), 1056-1071. https://doi.org/10.1016/j.peva.2011.07.015

[11] Brian D. Ondov, Todd J. Treangen, Páll Melsted, Adam B. Mallonee, Nicholas H. Bergman, Sergey Koren, and Adam M. Phillippy. 2016. Mash: fast genome and metagenome distance estimation using MinHash. Genome Biology 17, 1 (June 2016), 132. https://doi.org/10.1186/s13059-016-0997-x

[12] Rafail Ostrovsky and Yuval Rabani. 2007. Low Distortion Embeddings for Edit Distance. F. ACM 54, 5 (Oct. 2007). https://doi.org/10.1145/1284320.1284322 
[13] Mostofa Ali Patwary, Diana Palsetia, Ankit Agrawal, Wei-keng Liao, Fredrik Manne, and Alok Choudhary. 2012. A New Scalable Parallel DBSCAN Algorithm Using the Disjoint-set Data Structure. In Proceedings of the International Conference on High Performance Computing, Networking, Storage and Analysis (SC '12). IEEE Computer Society Press, Los Alamitos, CA, USA, 62:1-62:11. http://dl.acm.org/citation.cfm?id=2388996.2389081 event-place: Salt Lake City, Utah.

[14] Mostofa Ali Patwary, Diana Palsetia, Ankit Agrawal, Wei-keng Liao, Fredrik Manne, and Alok Choudhary. 2013. Scalable Parallel OPTICS Data Clustering Using Graph Algorithmic Techniques. In Proceedings of the International Conference on High Performance Computing, Networking, Storage and Analysis. ACM, New York, NY, USA, 49:1-49:12. https://doi.org/10.1145/2503210.2503255

[15] Jörg Sander, Martin Ester, Hans-Peter Kriegel, and Xiaowei Xu. 1998. DensityBased Clustering in Spatial Databases: The Algorithm GDBSCAN and Its Applications. Data Mining and Knowledge Discovery 2, 2 (June 1998), 169-194. https://doi.org/10.1023/A:1009745219419

[16] Erik L. L. Sonnhammer, Toni Gabaldón, Alan W. Sousa da Silva, Maria Martin, Marc Robinson-Rechavi, Brigitte Boeckmann, Paul D. Thomas, and Christophe Dessimoz. 2014. Big data and other challenges in the quest for orthologs. Bioinformatics 30, 21 (Nov. 2014), 2993-2998. https://doi.org/10.1093/bioinformatics/ btu492

[17] Martin Steinegger and Johannes Söding. 2018. Clustering huge protein sequence sets in linear time. Nature Communications 9, 1 (June 2018), 2542. https://doi. org/10.1038/s41467-018-04964-5

[18] Adam Szalkowski, Christian Ledergerber, Philipp Krähenbühl, and Christophe Dessimoz. 2008. SWPS3 - fast multi-threaded vectorized Smith-Waterman for IBM Cell/B.E. and $\times 86 /$ SSE2. BMC Research Notes 1, 1 (Oct. 2008), 107 . https: //doi.org/10.1186/1756-0500-1-107

[19] Richard Van Noorden, Brendan Maher, and Regina Nuzzo. 2014. The top 100 papers. Nature News 514, 7524 (Oct. 2014), 550. https://doi.org/10.1038/514550a

[20] Robert M. Waterhouse, Fredrik Tegenfeldt, Jia Li, Evgeny M. Zdobnov, and Evgenia V. Kriventseva. 2013. OrthoDB: a hierarchical catalog of animal, fungal and bacterial orthologs. Nucleic Acids Research 41, D1 (Jan. 2013), D358-D365. https://doi.org/10.1093/nar/gks1116

[21] Lucas D. Wittwer, Ivana Piližota, Adrian M. Altenhoff, and Christophe Dessimoz. 2014. Speeding up all-against-all protein comparisons while maintaining sensitivity by considering subsequence-level homology. Peerf 2 (Oct. 2014), e607. https://doi.org/10.7717/peerj.607 